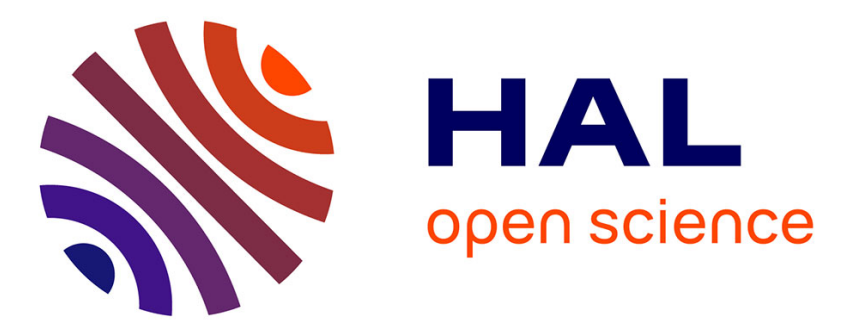

\title{
Molecular fingerprinting of from bone and joint infections
}

\author{
C. Luedicke, P. Slickers, R. Ehricht, S. Monecke
}

\section{To cite this version:}

C. Luedicke, P. Slickers, R. Ehricht, S. Monecke. Molecular fingerprinting of from bone and joint infections. European Journal of Clinical Microbiology and Infectious Diseases, 2010, 29 (4), pp.457463. 10.1007/s10096-010-0884-4 . hal-00570020

\section{HAL Id: hal-00570020 \\ https://hal.science/hal-00570020}

Submitted on 26 Feb 2011

HAL is a multi-disciplinary open access archive for the deposit and dissemination of scientific research documents, whether they are published or not. The documents may come from teaching and research institutions in France or abroad, or from public or private research centers.
L'archive ouverte pluridisciplinaire HAL, est destinée au dépôt et à la diffusion de documents scientifiques de niveau recherche, publiés ou non, émanant des établissements d'enseignement et de recherche français ou étrangers, des laboratoires publics ou privés. 


\title{
Molecular fingerprinting of Staphylococcus aureus from bone and joint infections
}

\author{
C. Luedicke • P. Slickers • R. Ehricht • S. Monecke
}

Received: 31 October 2009 /Accepted: 31 January 2010/Published online: 26 February 2010

(C) Springer-Verlag 2010

\begin{abstract}
The objective of the study was to determine if a clonal complex (CC) of Staphylococcus aureus or certain virulence and adhesion factors were associated with infections of bones and prosthetic implants. One hundred and nineteen isolates were characterised using microarrays. There was no evidence for a single virulence factor or CC being causative for bone and implant infections. Isolates belonged to 20 different CCs, with CC8 (19.33\%), CC45 $(17.65 \%)$ and CC30 (12.61\%) being dominant. Population structure and the relative abundances of virulence genes was similar to previously described isolates from healthy carriers. Differences to carrier isolates included a higher proportion of $\mathrm{CC} 45$, a lower proportion of $\mathrm{CC} 15$, as well as a higher abundance of sak (staphylokinase) among patient isolates. For 23 patients with infections of total knee or hip prosthetics, it was possible to simultaneously obtain nasal swabs. Fifteen (65.2\%) carried S. aureus in their anterior
\end{abstract}

Electronic supplementary material The online version of this article (doi:10.1007/s10096-010-0884-4) contains supplementary material, which is available to authorized users.

\section{Luedicke}

Medical Clinic and Policlinic I, University Hospital Dresden, Fetscherstrasse 74,

01307 Dresden, Germany

P. Slickers $\cdot$ R. Ehricht

CLONDIAG GmbH,

Loebstedter Strasse 103-105,

07749 Jena, Germany

\section{S. Monecke $(\square)$}

Institute for Medical Microbiology and Hygiene,

Faculty of Medicine "Carl Gustav Carus",

Technical University of Dresden,

Fetscherstrasse 74,

01307 Dresden, Germany

e-mail: monecke@rocketmail.com nares. In nine of them (39.1\%), isolates from the infection site were identical to carriage isolates. This suggests an elevated risk of infection for $S$. aureus carriers and the possibility of endogenous infection in a high proportion of them. Therefore, the pre-operative screening and eradication of $S$. aureus in patients receiving total joint prosthetics should be considered.

\section{Introduction}

Infections of bone tissue are a highly relevant issue in orthopaedics and trauma surgery. Due to comparatively poor blood circulation, infecting bacteria are well protected against the host immune defences, as well as against intravenously administered antibiotics. Thus, these pathogens are able to cause protracted or chronic infections, which can barely be cleared by the host and which pose a therapeutic problem. Additionally, the foci of infection can easily decrease the mechanical stability of the bone, resulting in pathological fractures. Other complications include secondary bacteraemia and sepsis or deformation and ankylosis of joints, resulting in permanent disability. An especially critical problem is the infection of joint endoprostheses. Because of the increasing average age of the population in industrialised countries, the number of patients carrying such prosthetics, including total knee arthroplasty (TKA) and total hip arthroplasty (THA), is steadily increasing. The infection of a TKA or THA is arguably one of the most difficult problems in orthopaedic surgery. For the patients, these infections mean a protracted illness with an uncertain outcome, while the hospital must deal with an incalculable economic risk.

While a variety of bacteria can cause bone infections, Staphylococcus aureus is one of their most relevant causes. 
It is common, with about $25 \%$ of the population being asymptomatic carriers. S. aureus carries a variety of different virulence and adhesion factors which might be of relevance in this kind of infection. It harbours, for example, superantigenic toxins, some of which are called enterotoxins, as they can also cause food poisoning. Isolates from osteomyelitis have been found to commonly produce enterotoxins [1]. Another superantigen, toxic shock syndrome toxin (encoded by tst 1), is known to cause septic polyarthritis in an animal model [2]. A recent study emphasised the role of a bi-component leukocidin (Panton-Valentine leukocidin, PVL) in cases of osteomyelitis and arthritis [3]. S. aureus also carries several different adhesion factors, so-called microbial surface components recognising adhesive matrix molecules of the host (MSCRAMMs, [4]). Because of the presence of a variety of toxins $[5,6]$, it can be regarded as more virulent than most other bacteria causing infections of bone and joint endoprostheses, such as coagulase-negative staphylococci. Its ability to form biofilms [7] and to persist in bone as metabolically less active small-colony variants [811], as well as its tendency to acquire drug resistance, are obstacles to effective antimicrobial chemotherapy. For these reasons, infections caused by $S$. aureus usually require aggressive therapy approaches, i.e. removal and delayed reimplantation [12], accompanied by long-term antimicrobial chemotherapy.

Because of the high number of bacterial genes involved, it would be extremely labourious to use polymerase chain reaction (PCR) in order to genotype clinical strain collections in detail. For that reason, we employed microarrays (StaphyType by CLONDIAG) to detect ca. 180 relevant genes (and alleles thereof) in patient isolates from the Dresden University Hospital, Germany. Thus, it was possible to simultaneously detect species markers, resistance genes, genes encoding exotoxins and adhesion factors, as well as $\mathrm{SCCmec}$, capsule and agr group typing markers. Furthermore, it was possible to assign the isolates to clonal complexes based on the analysis of hybridisation patterns and comparison to a database of reference strains which have been previously subjected to multi-locus sequence typing (MLST) [13, 14].

\section{Materials and methods}

\section{Bacterial isolates and DNA preparation}

One hundred and nineteen $S$. aureus isolates were obtained from routine diagnostics at the Dresden University Hospital, Germany. All aspirates and intraoperative swabs were considered, for which a diagnosis of osteomyelitis, Brodie abscess or of infections of TKA, THA or other implants (osteosynthesis with any metal plates, pins, rods, wires or screws) was determined. Isolates were collected consecu- tively in 2005-2006. Isolates from implant infections, for which parallel nasal swabs were taken, were obtained consecutively in 2008-2009. After the exclusion of copy strains (multiple, completely identical isolates from one individual patient), 119 isolates from 117 patients with a variety of bone infections were characterised. This included 46 cases of foreign body infections, two cases of infections of bone transplants, 14 intraosseous abscesses, as well as three cases of skin/soft tissue infections with involvement of the bone and ten cases of bursitis. For 26 patients with TKA or THA infections, it was possible to obtain additional nasal swabs. Sixteen of them yielded S. aureus. Details on the diagnosis and provenance for each single isolate are provided in the Electronic supplementary material.

$S$. aureus were cultured and subjected to conventional identification (Gram stain, catalase, coagulase, DNAse assays), as well as to biochemical profiling and susceptibility tests using an automated commercial system (Vitek 2, bioMérieux, Nuertingen, Germany). For microarray hybridisations, sub-cultures of single colonies were grown on Columbia blood agar and incubated overnight at $37^{\circ} \mathrm{C}$.

Lysis procedures and protocols have been described previously [13, 14]. In short, culture material was suspended in a lysis buffer (lysostaphin, lysozyme, ribonuclease A, Tris-HCl, Triton X-100 and water). After 45 min of incubation on a shaker, proteinase $\mathrm{K}$ and buffer $\mathrm{AL}$ (both from the DNeasy kit, QIAgen, Hilden, Germany) were added. After another incubation step, the sample was processed using the QIAgen tissue lysis kit. Finally, the quality of DNA samples was checked using concentration measurement at $260 \mathrm{~nm}$. Gel electrophoresis and ethidium bromide stain were performed to prove that DNA was neither fragmented nor contaminated with RNA.

\section{Array procedures}

The DNA array, as well as the protocols and procedures, have been previously described in detail [13, 14], and is now commercially available (StaphyType by CLONDIAG). The array covers 334 target sequences, which correspond to approximately 180 distinct genes and their allelic variants. Target genes (as well as hybridisation results) are listed in the Electronic supplementary material. The primer and probe sequences have been published previously $[13,14]$ or can be provided on request.

DNA samples were used as templates in a linear primer elongation using one primer per target. All targets were amplified simultaneously, and within this step, biotin-16dUTP was incorporated into the resulting amplicons. The amplicons were then hybridised to the microarray, followed by washing and blocking steps. Next, horseradishperoxidase-streptavidin conjugate was added. After the incubation and washing steps, hybridisations were visual- 
ised by adding a precipitating dye (Seramun Green, Seramun, Heidesee, Germany). Finally, an image of the microarray was taken and analysed using a designated reader and software script (CLONDIAG, Jena, Germany).

The affiliation of isolates to clonal complexes (CC) and/or to sequence types (ST) as defined by MLST [15] was determined by an automated comparison of hybridisation profiles to previously MLST-typed reference strains [13, 14]. Generally, the array allows the identification of clonal complex affiliations, but cannot discriminate sequence types, which differ in mutations affecting MLST genes (such as ST5 and ST225). However, sequence types which probably originate from gene transfer or hybridisation events can yield characteristic hybridisation patterns, allowing their discrimination. An example is ST239, which is derived from CC8 [16], but which yields a clearly discernible hybridisation pattern [14].

\section{Results}

\section{Affiliation to CCs and strains}

Isolates belonged to 20 different CCs (as defined by MLST [15]; http://saureus.mlst.net/). An overview of clonal complex affiliations is provided in Table 1.

The most common CC was CC8 (19.3\%, no MRSA, no PVL-positive isolates). Two of these 23 isolates displayed divergent hybridisation patterns. However, they belonged to sequence types which are part of CC 8 according to the MLST database (http://saureus.mlst.net/). One of these isolates yielded a hybridisation pattern as previously described [13, 14] for ST239, but lacked any SCCmec-associated genes. spa-typing of that isolate was not possible, since spa primers [17] failed to detect the spa gene region used for sequencing. The spa-specific probe did not yield a hybridisation signal. The other atypical CC8 isolate belonged to ST72, which differs from other $\mathrm{CC} 8$ in several features, such as the presence of the enterotoxin gene cluster egc (seg, sei, sem, sen, seo, seu and the presence of different alleles of several adhesion factors ( $f n b B, s a s G, s d r C, s d r D, v w b$ ).

The second most abundant CC was CC45 (17.6\%). This included 20 MSSA isolates and one ST45-MRSA-IV ("Berlin Epidemic MRSA"). There were no PVL-positive CC45 isolates.

Other common clonal complexes were CC30 (12.6\%, no MRSA, no PVL), CC101 (7.6\%, no MRSA, no PVL) and CC5 (6.7\%, no PVL-positive isolates but including one isolate of ST228-MRSA-I, "South German Epidemic MRSA" and three isolates of CC5-MRSA-II, "Rhine-Hesse Epidemic MRSA/UK-EMRSA-3").

All other isolates belonged to a total of 15 different CCs, with all of them comprising less than $7 \%$ of isolates
(Table 1). Two of these isolates were MRSA, belonging to ST22-MRSA-IV ("Barnim Epidemic MRSA/UK-EMRSA15") and ST80-MRSA-IV ("European community acquired MRSA clone"), respectively.

The four PVL-positive isolates belonged to CC22MSSA, ST80-MRSA-IV, ST96-MSSA and CC121-MSSA. The tst 1 -positive isolates belonged to either CC30-MSSA (12 isolates) or ST426-MSSA (two isolates).

\section{Virulence factors}

The complete microarray hybridisation data are provided as a supplementary file.

The most abundant enterotoxin genes were those of the enterotoxin gene cluster egc (seg, sei, sem, sen, seo, seu), which were present in 62 out of 119 isolates (52.1\%). Other common virulence factors included the genes encoding enterotoxins $\mathrm{C}$ and $\mathrm{L}$ ( $\mathrm{sec}$ and sel; in 20 isolates, $16.8 \%$ ), enterotoxin B ( $\mathrm{seb}$; ten isolates, $8.4 \%$ ), toxic shock syndrome toxin (in 14 isolates, 11.8\%) and an enterotoxin homologue (ORF CM14, GenBank U10927.2, in 11 isolates, 9.2\%).

Most isolates carried beta-haemolysin integrating phages, with the beta-haemolysin gene $h l b$ being truncated or interrupted in 115 isolates (96.6\%). Among phage-borne genes, staphylokinase ( $a k$ ) was the most common, with 108 (90.8\%) being positive. Two distinct alleles of enterotoxin A were found (sea in 16 isolates, 13.4\%, and sea-N315 in 14 isolates, $11.8 \%$ ).

PVL genes were found in four isolates (3.4\%). These patients suffered from a phlegmone of the knee, a postoperative abscess of the femur, a knee empyema after contact with family members carrying the same PVLMRSA and from bursitis, respectively.

The bi-component leukocidin homologue lukD+lukE was found in 75 isolates $(63.0 \%)$. However, isolates of CC15, CC101 and ST426 yielded signals with only one out of both components, which might be indicative of the presence of deviant, yet unsequenced alleles. CC9, CC30, CC45 and CC59 were generally negative for $l u k D+l u k E$, as well as the single isolate of ST228-MRSA-I.

The ACME locus (arginine metabolic locus, $\operatorname{arc} A, \operatorname{arc} B$, $\operatorname{arc} C, \operatorname{arcD})$ was found in three isolates $(2.5 \%)$, all of them being CC8-MSSA. Exfoliative toxins were rare (etA in three isolates, $2.5 \%$, and $e t D$ in nine isolates, $7.6 \%$ ). These isolates were not associated with symptoms of the scalded skin syndrome. et $D$ was always accompanied by edinB (epidermal cell differentiation inhibitor B), being detectable in all $\mathrm{CC} 25$ and $\mathrm{CC} 80$ isolates.

Biofilm genes and capsule types

Biofilm-related genes $i c A, i c C$ and $i c D$ were detected in all isolates, whereas another biofilm-associated gene, bap, was 
Table 1 Clonal complex and strain affiliations of tested Staphylococcus aureus isolates

\begin{tabular}{|c|c|c|c|}
\hline Clonal complex & Strain designation based on microarray hybridisation & Number of isolates & Percentage \\
\hline \multirow[t]{3}{*}{$\mathrm{CC} 8$} & CC8-MSSA & 21 & 17.65 \\
\hline & ST72-MSSA & 1 & 0.84 \\
\hline & ST239-MSSA & 1 & 0.84 \\
\hline \multirow[t]{2}{*}{$\mathrm{CC} 45$} & CC45-MSSA & 20 & 16.81 \\
\hline & ST45-MRSA-IV & 1 & 0.84 \\
\hline $\mathrm{CC} 30$ & CC30-MSSA & 15 & 12.61 \\
\hline $\mathrm{CC} 101$ & CC101-MSSA & 9 & 7.56 \\
\hline \multirow[t]{3}{*}{$\mathrm{CC} 5$} & CC5-MSSA & 4 & 3.36 \\
\hline & ST5-MRSA-II & 3 & 2.52 \\
\hline & ST228-MRSA-I & 1 & 0.84 \\
\hline $\mathrm{CC} 25$ & CC25-MSSA & 8 & 6.72 \\
\hline $\mathrm{CC} 15$ & CC15-MSSA & 7 & 5.88 \\
\hline $\mathrm{CC} 12$ & CC12-MSSA & 6 & 5.04 \\
\hline \multirow[t]{2}{*}{$\mathrm{CC} 22$} & CC22-MSSA & 3 & 2.52 \\
\hline & ST22-MRSA-IV & 1 & 0.84 \\
\hline $\mathrm{CC} 7$ & CC7-MSSA & 3 & 2.52 \\
\hline $\mathrm{CC} 121$ & CC121-MSSA & 3 & 2.52 \\
\hline CC9 & CC9-MSSA & 2 & 1.68 \\
\hline CC49 & CC49-MSSA & 2 & 1.68 \\
\hline ST426 & ST426-MSSA & 2 & 1.68 \\
\hline $\mathrm{CC} 20$ & CC20-MSSA & 1 & 0.84 \\
\hline CC59 & CC59-MSSA & 1 & 0.84 \\
\hline $\mathrm{CC} 80$ & ST80-MRSA-IV & 1 & 0.84 \\
\hline $\mathrm{CC} 88$ & ST88-MSSA & 1 & 0.84 \\
\hline CC96/154 & ST96-MSSA & 1 & 0.84 \\
\hline CC97 & CC97-MSSA & 1 & 0.84 \\
\hline
\end{tabular}

always absent. The more predominant capsule type was 8 (capH8, capI8, capJ8, capK8). It was found in 71 isolates (59.7\%) belonging to CC7, CC12, CC15, CC30, CC45, CC59, ST80, CC88, ST96, CC101, CC121, ST239 and ST426. All remaining isolates belonged to capsule type 5 . Capsule type 1 was not detected.

\section{MSCRAMM genes}

The presence of two MSCRAMM genes, cna (encoding the collagen adhaesin) and $\operatorname{sas} G$ (gene for $S$. aureus surface protein G), showed a clear relation to clonal complex affiliations. cna was detected in 52 isolates (43.7\%), which all belonged to CC12, CC22, CC30, CC45, CC96, CC121, ST239 and ST426. Except for a single cna-negative ST426 isolate, all isolates of these clonal complexes were positive for this gene. sas $G$ was present in 50 isolates $(42.4 \%)$. It exclusively occurred in CC5, CC8, CC15, CC22, ST49, CC59, ST80, CC88 and ST96.

All other MSCRAMM genes ( $b b p, c l f A, c l f B, e b h, e b p S$, eno, fib, fnbA, fnbB, map, $s d r C, s d r D$ and $v w b$ ) essentially were found in all clonal complexes. However, in many cases, allelic variants could be recognised which were specific for certain clonal complexes. In a small number of isolates, deletions occurred. For instance, $b b p$ was absent from a small number of $\mathrm{CC} 12, \mathrm{CC} 25, \mathrm{CC} 30$ and $\mathrm{CC} 45$ isolates, although it was detectable in a majority of isolates belonging to these clonal groups.

\section{Resistance genes}

The most abundant resistance marker was the beta-lactamase operon (blaZ, blaI, blaR), which was present in $64.7 \%$ of isolates. SCCmec elements were rare; seven isolates $(5.9 \%)$ were mecA-positive and, phenotypically, methicillinresistant. Interestingly, five methicillin-susceptible isolates carried $c c r A / B-4$ genes without being mecA-positive (CC8). Erythromycin/clindamycin resistance genes erm $A$ and erm $C$ were present in seven $(5.9 \%)$ or 11 isolates $(9.2 \%)$, respectively. Genes encoding aminoglycoside-modifying enzymes were rare. The gene responsible for gentamicin/tobramycin resistance, aacA-aphD, was found in four isolates $(3.4 \%)$, 
one of them being an MRSA (ST228-MRSA-I). The tobramycin resistance gene $a a d D$ was detected in six isolates $(5.0 \%)$, including two isolates of the CC5-MRSA-II. The genes aphA-3+sat (a transposon encoding resistance towards neo-/kanamycin and streptothricin) was present in three isolates (ST45-MRSA-IV, ST228-MRSA-I and ST80MRSA-IV). Other rare resistance determinants included tet $M$ (in two isolates, 1.7\%), tet $K$ (four isolates, 3.4\%) and farl (one isolate, $0.8 \%$, ST80-MRSA-IV).

Comparison of TKA or THA isolates to nasal swab isolates

For 23 patients with S. aureus infections of total knee or hip prosthetics, it was possible to obtain nasal swabs in order to detect and type possible $S$. aureus carriage strains (Table 2). Fifteen of them $(65.2 \%)$ carried S. aureus in their anterior nares.

Five patients $(21.7 \%)$ had one strain in the arthroplasty, but another in the nasal swab. The arthroplasty of one patient was infected with two CC25 strains which, however, were identical in all markers except for the betalactamase operon. This patient carried a completely different strain in the nose.

In eight patients (34.8\%), strains from the infection site were identical to the carriage isolates. In one additional case, two strains (CC8- and CC97-MSSA) were found at the site of the infection. The CC8-MSSA was identical to one out of two different CC8-MSSA strains also found in his nasal swab.

\section{Discussion}

When genotyping $S$. aureus isolates from bone and prosthetics infections, a wide variety of exotoxin genes and virulence factors was detected. While the staphylokinase gene sak and the enterotoxin gene cluster egc were present in a majority of isolates, none of the "classical" enterotoxin genes or PVL were conspicuously common.

In order to assess the significance of virulence factors, their relative abundances were compared to data on $S$. aureus isolates obtained from healthy carriers from the same geographical area [18]. Clear differences affected only staphylokinase (sak), PVL genes and enterotoxin B (seb). The gene encoding staphylokinase (sak) was detected in $71.6 \%$ of carriers [18] and in $90.8 \%$ of patients. It was found in $83 \%$ of skin and soft tissue infection isolates [19]. Thus, the presence of sak might be related to a higher invasiveness of $S$. aureus in humans. Staphylokinase forms complexes with plasminogen, resulting in the formation of active plasmin which, as a proteolytic enzyme, might, indeed, facilitate the entry of staphylococci into the deeper host tissues [20]. Interestingly, sak is situated on a prophage which integrates into the beta-haemolysin gene [21-23]. Therefore, infection of an $S$. aureus isolate with a phage has an impact on virulence and plays a role in determining the course of the interaction of $S$. aureus with its human host. By the transmission of $s a k$, the phage apparently compensates for any adverse effects it might cause, such as the inactivation of the beta-haemolysin. PVL was detected in $0.65 \%$ of carriers [18], but in $3.36 \%$ of patients. This means that it is associated with clinical disease but it is, by far, too rare to be the sole significant factor in the pathogenesis of bone infections. It is known to be highly associated with skin and soft tissue infections [24], being detectable in as much as $30 \%$ of abscess isolates [19]. Indeed, the four patients with PVL-positive isolates from the present study had soft tissue infections encroaching the bone. The enterotoxin B gene seb was found in $3.9 \%$ of carriage [18] but in $8.4 \%$ of patient isolates. This could also mean that it was associated with disease, but was too rare to be the sole factor responsible. Concerning other virulence factors as well as capsule types, differences to carrier isolates [18] were insignificant. Some enterotoxins were slightly more common in patients (sea, egc, ORF CM14), whereas others were more prevalent in carriage isolates (tstl, sed $+s e j+s e r)$. Several MSCRAMM genes were also more common among healthy carriers (bbp, sas $G, s d r D)$.

A wide range of different clonal complexes of $S$. aureus was found to be involved in bone infections. Altogether, 20 different CCs were detected. When comparing CC affiliations of bone isolates to typing data on $S$. aureus isolates obtained from healthy carriers from the same geographic area [18], generally, a similar pattern can be observed. CC8 and $\mathrm{CC} 30$ dominate in both populations. $\mathrm{CC} 15$, which was very common $(16.77 \%,[18])$ among healthy carriers, was rather rarely detected in bone infections $(5.88 \%)$. This could indicate a comparatively low virulence or a lesser potential to cause invasive infection. Noteworthy is that not a single $\mathrm{CC} 15$ isolate was found to carry sak. On the other hand, CC45 was more common among bone infections than among healthy carriers [18]. Since there was no obvious difference in toxin carriage, this observation could theoretically be related to the presence of a gene or gene cluster which was not yet covered by the DNA array. Another explanation could be a different regulation or expression of a virulence factor which was also found in other strains. This could be clarified by expression studies. Another possibility could be a difference in physiology, such as a better ability to cope with anaerobic conditions or to form small colony variants (SCV). Thus, it would be interesting to type known SCV lines in order to determine the prevalence of $\mathrm{CC} 45$. A potentially higher invasiveness of CC45 deserves further study, especially because there are important epidemic MRSA strains from that lineage (CC45MRSA-II, CC45-MRSA-IV). 
The similarity of population structures of $S$. aureus isolates from healthy carriers [18] and patients suggests either an endogenous infection or (in the case of surgical patients) an iatrogenic transmission. A local transmission of skin flora during the surgical procedure might play a role in, e.g. S. epidermidis infections, but $S$. aureus usually does not colonise intact skin [25]. When typing isolates from total knee or hip prosthetics in parallel to nasal swab isolates, in as much as $65 \%$ of these patients, a nasal colonisation with $S$. aureus was found. This is a higher proportion than in a normal population (about 30\%, see [26]). Approximately half of these carriers had the same strain in the site of the infection as in their anterior nares. Therefore, we assume that a low-level bacteraemia originating from colonised body sites might contribute to endogenous infections, especially under stressful conditions such as during major surgery. However, a high proportion of patients did not carry S. aureus at all, or they had other strains in their nares than at the infection site. It would be premature to assume that all of these infections originated from the colonising flora of the surgical staff. This might certainly happen in some cases, as in patient O-109 from
Table 2, who had a typical hospital-associated MRSA strain in an arthroplasty infection (ST5-MRSA-II, which is one of the predominant strains in the region where the study was conducted [13]). The high proportion of $S$. aureus carriers among patients with TKA/THA infections might indicate that these people could more easily be colonised or infected by $S$. aureus than the average population. They also could carry multiple strains, which might not have all been detected by taking a single nasal swab, or they might be colonised at other body sites. In order to resolve this issue, it would be necessary to follow a large patient population over a rather long time-span and to obtain multiple and repeated screening samples of patients, as well as of surgical staff. Since TKA/THA infections are not that common, a prospective study would be extremely elaborate and time-consuming. An easier option would probably be just to intervene pre-emptively. These measures would include the detection and eradication $[27,28]$ of any $S$. aureus carriage among patients prior to orthopaedic surgery. Since $S$. aureus carriers appear to be especially at risk, the prolongation of perioperative antibiotic prophylaxis and the use of additional techniques for decreasing the

Table 2 Parallel typing of arthroplasty and nasal swab isolates

\begin{tabular}{|c|c|c|}
\hline Patient ID & Arthroplasty & Nasal swab \\
\hline O-103 & CC20-MSSA & CC5-MSSA \\
\hline O-105 & CC22-MSSA & Identical CC22-MSSA \\
\hline O-106 & CC25-MSSA and CC15-MSSA & No $S$. aureus detectable \\
\hline O-107 & CC8-MSSA & Identical CC8-MSSA \\
\hline O-108 & CC8-MSSA & Identical CC8-MSSA \\
\hline O-109 & ST5-MRSA-II & CC30-MSSA \\
\hline O-110 & CC8-MSSA & Identical CC8-MSSA \\
\hline O-111 & CC15-MSSA & Identical CC15-MSSA \\
\hline $\mathrm{O}-112$ & CC45-MSSA & Identical CC45-MSSA \\
\hline O-113 & $\begin{array}{l}\text { Two different CC25-MSSA isolates } \\
\text { (differing only in presence of blaZ operon) }\end{array}$ & CC8-MSSA \\
\hline O-114 & $\mathrm{CC} 101$ & Identical CC101 \\
\hline O-115 & CC97-MSSA and CC8-MSSA & $\begin{array}{l}\text { Two different CC8-MSSA, one of them identical } \\
\text { to the CC8-MSSA from the arthroplasty }\end{array}$ \\
\hline O-116 & CC8-MSSA & No $S$. aureus detectable \\
\hline O-117 & ST49 & Identical ST49 \\
\hline O-118 & CC7 & ST5-MRSA-II \\
\hline O-119 & CC8-MSSA & No $S$. aureus detectable \\
\hline O-120 & $\mathrm{CC} 30$ & No $S$. aureus detectable \\
\hline O-121 & $\mathrm{CC} 72$ & No $S$. aureus detectable \\
\hline $\mathrm{O}-122$ & $\mathrm{CC} 12$ & No $S$. aureus detectable \\
\hline $\mathrm{O}-123$ & CC15-MSSA & No $S$. aureus detectable \\
\hline O-124 & CC45-MSSA & CC8-MSSA \\
\hline O-126 & CC45-MSSA & No $S$. aureus detectable \\
\hline O-127 & CC30-MSSA & CC22-MSSA \\
\hline
\end{tabular}


risk of infection (adhesive incise drape, jet lavage, laminar flow etc.) should be considered. Finally, staff surveillance should target not only MRSA, but any $S$. aureus.

Acknowledgements The authors thank A. Ruppelt, H. Kanig, S. Kolewa, E. Müller, I. Engelmann and J. Sachtschal for their excellent technical assistance, as well as R. Schuster and T. Uhlig for developing the software used in the microarray analysis. We acknowledge Prof. E. Jacobs, Prof. G. Ehninger and E. Ermantraut for their support. We thank D. Rowe and W. Rudolph for critically reading the manuscript.

The study was funded by the Vice-Directorate for Research of the Faculty of Medicine "Carl Gustav Carus" (MedDrive program 2008). Ralf Ehricht and Peter Slickers are employees of CLONDIAG.

\section{References}

1. Sourek J, Výmola F, Trojanová M, Zelenková L, Matějovska V, Bergdoll MS (1979) Enterotoxin production by Staphylococcus aureus strains isolated from cases of chronic osteomyelitis. J Clin Microbiol 9:266

2. Cunningham R, Cockayne A, Humphreys H (1996) Clinical and molecular aspects of the pathogenesis of Staphylococcus aureus bone and joint infections. J Med Microbiol 44:157

3. Dohin B, Gillet Y, Kohler R, Lina G, Vandenesch F, Vanhems P, Floret D, Etienne J (2007) Pediatric bone and joint infections caused by Panton-Valentine leukocidin-positive Staphylococcus aureus. Pediatr Infect Dis J 26:1042

4. Clarke SR, Foster SJ (2006) Surface adhesins of Staphylococcus aureus. Adv Microb Physiol 51:187

5. Dinges MM, Orwin PM, Schlievert PM (2000) Exotoxins of Staphylococcus aureus. Clin Microbiol Rev 13:16

6. Kaneko J, Kamio Y (2004) Bacterial two-component and heteroheptameric pore-forming cytolytic toxins: structures, pore-forming mechanism, and organization of the genes. Biosci Biotechnol Biochem 68:981

7. Brady RA, Leid JG, Calhoun JH, Costerton JW, Shirtliff ME (2008) Osteomyelitis and the role of biofilms in chronic infection. FEMS Immunol Med Microbiol 52:13

8. Swingle EL (1935) Studies on small colony variants of Staphylococcus aureus. J Bacteriol 29:467

9. Proctor RA, Balwit JM, Vesga O (1994) Variant subpopulations of Staphylococcus aureus as cause of persistent and recurrent infections. Infect Agents Dis 3:302

10. von Eiff C, Bettin D, Proctor RA, Rolauffs B, Lindner N, Winkelmann W, Peters G (1997) Recovery of small colony variants of Staphylococcus aureus following gentamicin bead placement for osteomyelitis. Clin Infect Dis 25:1250

11. von Eiff C, Proctor RA, Peters G (2000) Staphylococcus aureus small colony variants: formation and clinical impact. Int J Clin Pract Suppl 44

12. Brandt CM, Duffy MC, Berbari EF, Hanssen AD, Steckelberg JM, Osmon DR (1999) Staphylococcus aureus prosthetic joint infection treated with prosthesis removal and delayed reimplantation arthroplasty. Mayo Clin Proc 74:553

13. Monecke S, Jatzwauk L, Weber S, Slickers P, Ehricht R (2008) DNA microarray-based genotyping of methicillin-resistant Staph- ylococcus aureus strains from Eastern Saxony. Clin Microbiol Infect 14:534

14. Monecke S, Slickers P, Ehricht R (2008) Assignment of Staphylococcus aureus isolates to clonal complexes based on microarray analysis and pattern recognition. FEMS Immunol Med Microbiol 53:237-251

15. Enright MC, Day NPJ, Davies CE, Peacock SJ, Spratt BG (2000) Multilocus sequence typing for characterization of methicillinresistant and methicillin-susceptible clones of Staphylococcus aureus. J Clin Microbiol 38:1008

16. Robinson DA, Enright MC (2004) Evolution of Staphylococcus aureus by large chromosomal replacements. J Bacteriol 186:1060

17. Harmsen D, Claus H, Witte W, Rothgänger J, Claus H, Turnwald D, Vogel U (2003) Typing of methicillin-resistant Staphylococcus aureus in a University Hospital setting by using novel software for spa repeat determination and database management. J Clin Microbiol 41:5442

18. Monecke S, Luedicke C, Slickers P, Ehricht R (2009) Molecular epidemiology of Staphylococcus aureus in asymptomatic carriers. Eur J Clin Microbiol Infect Dis 28:1159

19. Monecke S, Slickers P, Ellington M, Kearns A, Ehricht R (2007) High diversity of Panton-Valentine leukocidin-positive, methicillin-susceptible isolates of Staphylococcus aureus and implications for the evolution of community-associated methicillin-resistant $S$. aureus. Clin Microbiol Infect 13:1157

20. Bokarewa MI, Jin T, Tarkowski A (2006) Staphylococcus aureus: Staphylokinase. Int J Biochem Cell Biol 38:504

21. van Wamel WJB, Rooijakkers SHM, Ruyken M, van Kessel KPM, van Strijp JAG (2006) The innate immune modulators staphylococcal complement inhibitor and chemotaxis inhibitory protein of Staphylococcus aureus are located on \{beta\}-hemolysin-converting bacteriophages. J Bacteriol 188:1310

22. Coleman D, Knights J, Russell R, Shanley D, Birkbeck TH, Dougan G, Charles I (1991) Insertional inactivation of the Staphylococcus aureus beta-toxin by bacteriophage phi 13 occurs by site- and orientation-specific integration of the phi 13 genome. Mol Microbiol 5:933

23. Coleman DC, Sullivan DJ, Russell RJ, Arbuthnott JP, Carey BF, Pomeroy HM (1989) Staphylococcus aureus bacteriophages mediating the simultaneous lysogenic conversion of beta-lysin, staphylokinase and enterotoxin A: molecular mechanism of triple conversion. J Gen Microbiol 135:1679

24. Lina G, Piémont $\mathrm{Y}$, Godail-Gamot F, Bes M, Peter MO, Gauduchon V, Vandenesch F, Etienne J (1999) Involvement of Panton-Valentine leukocidin-producing Staphylococcus aureus in primary skin infections and pneumonia. Clin Infect Dis 29:1128

25. Larson EL, Hughes CA, Pyrek JD, Sparks SM, Cagatay EU, Bartkus JM (1998) Changes in bacterial flora associated with skin damage on hands of health care personnel. Am J Infect Control $26: 513$

26. van Belkum A, Melles DC, Nouwen J, van Leeuwen WB, van Wamel W, Vos MC, Wertheim HFL, Verbrugh HA (2009) Coevolutionary aspects of human colonisation and infection by Staphylococcus aureus. Infect Genet Evol 9:32

27. Crossley K (2009) Review: intranasal mupirocin ointment reduces Staphylococcus aureus infections in nasal carriers. Evid Based Med 14:110

28. van Rijen M, Bonten M, Wenzel R, Kluytmans J (2008) Mupirocin ointment for preventing Staphylococcus aureus infections in nasal carriers. Cochrane Database Syst Rev CD006216 